\title{
Laser induced THz Sommerfeld waves along metal wire
}

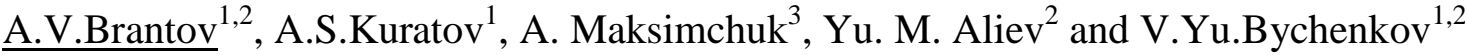 \\ ${ }^{1}$ Dukhov Research Institute of Automatics (VNIIA), Moscow, Russia, brantov@ lebedev.ru \\ ${ }^{2}$ P.N. Lebedev Physics Institute, Russian Academy of Sciences, Moscow, Russia \\ ${ }^{3}$ Center for Ultrafast Optical Science, University of Michigan, Ann Arbor, USA
}

The thin metal wires can be utilized for guiding electron bunches $[1,2]$ as well as surface waves [3] during generation from irradiation of a wire target by short relativistically intense laser pulse. In such interaction a strong impulse type Thz surface wave can be excited at the metal surface due to a current of the high energy escaping electrons and electric sheath formation. These waves may propagate along wire without strong damping in the form of Sommerfeld wave. In this paper the electron bunch guiding along wire and supported by strong Sommerfeld surface wave is modeled and compared with experimental results.

To describe the initial stage of electron bunch formation and electromagnetic surface wave formation we have performed PIC simulation of laser-wire interaction considering a target as a plasma cylinder with given plasma density. The 3D simulations have been performed by using 3D PIC code Mandor and commercial code VSim (Vorpal). As example, we illustrate our findings with the following parameters. A linearly polarized laser pulse with maximum intensity of $(1-3) \times 10^{19} \mathrm{~W} \mathrm{~cm}^{-2}$ has a Gaussian shape in both the temporal (FWHM duration $\tau_{0}=50 \mathrm{fs}$ ) and transverse dimensions (FWHM focal spot size $d=4$ $\mu \mathrm{m})$. The maximum intensity corresponds to dimensionless laser field amplitude $a_{0}=(2.7-4.7)$ for laser wavelength $\lambda=1 \mu \mathrm{m}$. The laser is focused at the front surface of a cylindrical plasma target (with the axis along Y) with diameter of $8 \mu \mathrm{m}$ consisting of electrons and heavy ions $\left(Z m_{i} / m_{e}=4 \times 1836\right)$. The plasma density is $5 n_{c}$ ( $n_{c}$ is the electron critical density) inside interaction aria (cylinder piece of $10 \mu \mathrm{m}$ length from the focal spot center in both directions) and $2 n_{c}$ outside. The simulation box has the size $x \times y \times z=$ $20 \mu \mathrm{m} \times 200 \mu \mathrm{m} \times 20 \mu \mathrm{m}$ and the spatial resolution is $\Delta$, $x \times \Delta y \times \Delta z=0.02 \mu \mathrm{m} \times 0.1 \mu \mathrm{m} \times 0.05 \mu \mathrm{m}$.

The electron density after irradiation of a wire by laser pulse focused in the wire center is presented in Fig.1. Two symmetrical electron bunches propagated along wire from interaction area with velocity of the order of speed of light are clearly seen. Under the action of laser pulse, significant number of electrons inside a hot spot is heated to the high, multi-MeV, energies. The most of them form a sheath field electrostatic structure around wire as uncompensated positive target charge prevented them from escaping. At the same time, these electrons propagates along wire increasing a heated target surface, the length, $L_{0}$, of which is defined by the initial focal spot size, laser pulse duration and characteristic electron cooling time, i.e. $L_{0} \sim d+c\left(\tau_{0}+\tau_{c}\right)$ [4]. This heated area size roughly coincides with electron bunch longitudinal size, which is formed after a laser pulse termination.
Because of the symmetry of electron bunch generation, several simulation runs have been performed with laser pulse, which was focused near the wire edge. It has been confirmed that the electron bunch length increases with laser pulse duration reaching the value $L_{0} \sim c \tau_{0}$ for $\tau_{0}>300 \mathrm{fs}$. The simulations show that all electrons forming a bunch originate from the laser-target interaction area and electrons moving along a wire are accompanied by the propagating surface electromagnetic wave (see Fig. 2). The amplitude of this electromagnetic wave is much high as compared to the intrinsic electron bunch filed.

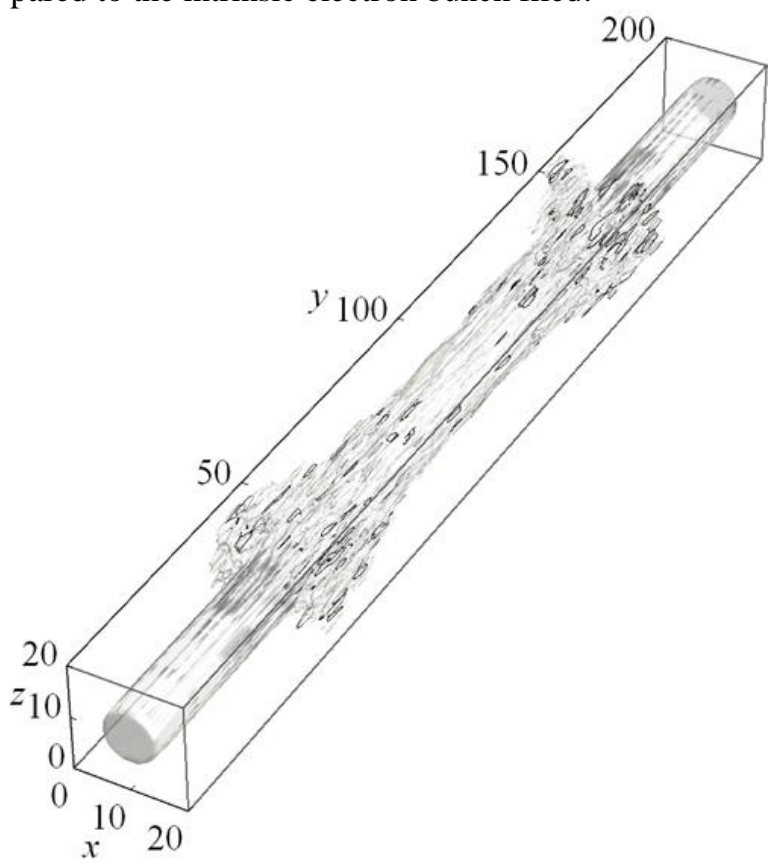

Fig. 1. Electron density contour plot at instant of $350 \mathrm{fs}$ after wire irradiation by laser pulse with $a_{0}=2.7$.

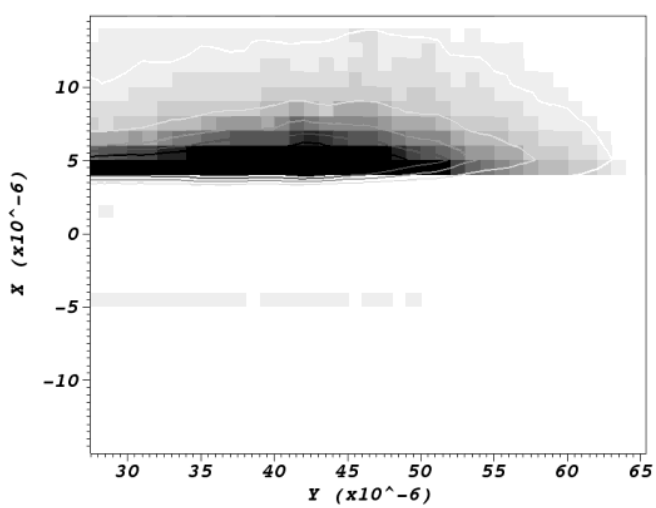

Fig. 2. Transversal $(x>0)$ and longitudinal $(x<0)$ electric field distributions in $x-y$ plane at the instant of 350 fs after wire irradiation by laser pulse with $a_{0}=4.7$. Wire lies be- 
tween $\mathrm{x}=-4$ and $\mathrm{x}=4$ and a laser pulse is focused to $\mathrm{y}=$ $10 \mu \mathrm{m}$.

The theory of Sommerfeld surface wave generation due to current of heated electrons has been developed. Based on solution of Maxwell equations the surface wave profile in a wave zone (far field) has been calculated. Expression for the magnetic field outside a wire with the radius $R$ generated due to the radial electron current density, $j_{r}$, has the following form

$B^{s}=\frac{2}{c R} \int \frac{d \omega k^{s} K_{1}\left(k_{+}^{s} r\right)}{k_{+}^{s 2} d{ }^{\prime}\left(k^{s}, \omega\right)} \mathrm{e}^{i\left(\omega t \pm k^{s} z\right)} \int_{R}^{\infty} d x \frac{x K_{1}\left(k_{+}^{s} x\right)}{K_{0}^{2}\left(k_{+}^{s} R\right)} j_{r}(x)$

Here $k_{+}{ }^{2}=k^{2}-\omega^{2} / \mathrm{c}^{2}, k_{-}^{2}=k^{2}-\varepsilon \omega^{2} / \mathrm{c}^{2}, \varepsilon$ is wire dielectric permittivity, $I_{(0,1)}, K_{(0,1)}$ are the modified Bessel functions and $k^{s}$ is a solution of the standard dispersion relation for Sommerfeld wave $d=0$, where

$d(k, \omega)=\frac{K_{1}\left(k_{+} R\right)}{k_{+} K_{0}\left(k_{+} R\right)}+\frac{\varepsilon I_{1}\left(k_{-} R\right)}{k_{-} I_{0}\left(k_{-} R\right)}$

As a radial current we have used both the current of the electrons forever leaving a target and the current of hot electrons forming a sheath structure. We assumed the Gaussian current profile along the wire with characteristic length of $L_{0}$, as well as the temporal Gaussian profile (with characteristic time scale of $\left.\tau_{0}\right)$. We also assumed that heated electrons move in $r$ direction with speed of light and have exponential distribution with characteristic scales of their Debay length, $r_{\text {De }}$. Density of the hot electrons can be estimated from energy balance, assuming 30\% laser energy deposition to the electrons, an average hot electron energy from simple ponderomotive scaling $a_{0} m_{e} c^{2}$ and the current volume $\sim L_{0} 2 \pi R r_{\mathrm{De}}$. Some of the electrons may overcome potential barrier and escape from the target. Escaping electron density depends on magnitude of the electrostatic potential and can be estimated as $\sim a_{0} m_{e} c^{2} / 2 \pi e^{2} R^{2}$. Based on the above estimates, one can calculate a polar magnetic field of the surface wave (see. Fig. 3). We conclude, that electron current due to the escaping electrons and due to those, which form a sheath give comparable contribution to Sommerfeld wave generation.

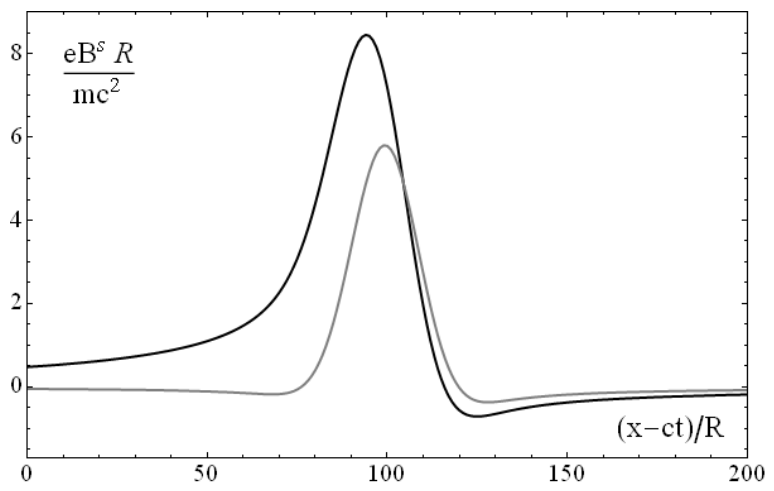

Fig. 3. Surface magnetic field at the tungsten wire boundary $(r=R=25 \mu \mathrm{m})$ for the laser pulse with $a_{0}=4.7, \mathrm{~d}=4 \mu \mathrm{m}$ and $\tau_{0}=400 \mathrm{fs}$, generated due to the escaping electrons (black curve) and the sheath forming electrons (gray curve).

To conclude, the numerical simulations performed have shown a formation of relativistic electron bunches propagating along the wire together with electromagnetic surface waves in the process of wire target irradiation by short relativistically intense laser pulse.

This work was supported by the Russian Foundation for Basic Research (grants No 16-02-00088, 1802-00452).

\section{References}

1. Nakajima H., Tokita S., Inoue S., Hashida M., Sakabe $S$. Divergence-free transport of laser-produced fast electrons along a meter-long wire target// Phys. Rev. Lett. 2013. V. 110. P. 155001

2. A. Maksimchuk, P. Belancourt, M. J.-E. Manuel et al Study of surface current confinement in high-intensity laser interactions with wire targets // Bull. Am. Phys. Soc. 2013. V. 58. P. 242; A. Maksimchuk, P. Belancourt, P. Kordell et al., Guiding of high-energy electrons in high-intensity-laser interactions with wire targets through surface wave excitation // Bull. Am. Phys. Soc. 2014. V. 59, No. 15, NP8.00101

3. Tokita S., Sakabe S., Nagashima T., Hashida M., Inou $S$. Strong sub-terahertz surface waves generated on a metal wire by high-intensity laser pulses // Sci. Rep. 2015 V. 5. P. 8268.

4. Kuratov A.S., Brantov A.V., Aliev Yu.M., Bychenkov $V . Y u$. Laser-induced thermoelectric current as a source of generation of $\mathrm{THz}$ surface electromagnetic waves // Quantum Electronics 2018 V.48. No. 7. P. 653. 